\title{
Gastrointestinal toxicities associated with immune checkpoint inhibitors: a disproportionality analysis leveraging VigiBase, the WHO Adverse Drug Reaction Database
}

\author{
Sifu HUANG ${ }^{1 *}$, Xuefeng BAI ${ }^{2 *}$, Taiyong FANG ${ }^{1}$, Yanta GUO ${ }^{1}$, Kainan $\mathrm{ZHENG}^{4}$, Xiahong $\mathrm{LIN}^{2,3 凶}$ \\ ${ }^{I}$ Department of Gastroenterology, the Second Affiliated Hospital of Fujian Medical University, Quanzhou 362000, China \\ ${ }^{2}$ Department of Endocrinology, the Second Affiliated Hospital of Fujian Medical University, Quanzhou 362000, China \\ ${ }^{3}$ Department of Medical Administration, the Second Affiliated Hospital of Fujian Medical University, Quanzhou 362000, China \\ ${ }^{4}$ IT Application Department, Xiamen C\&D Corporation Limited, Xiamen 361000, China
}

With the improvement of people's living standards, gastrointestinal adverse reactions caused by various adverse factors have attracted more and more people's attention. A recent study has indicated that coronavirus disease 2019 (COVID-19) could also invade the gastrointestinal tract, leading to gastrointestinal adverse reactions (Song et al., 2020). In recent years, immunotherapy has provided certain effects for some patients with advanced malignant tumors. A microencapsulation of immunoglobulin $\mathrm{Y}(\mathrm{IgY})$ was reported to provide an effective way to preserve $\operatorname{IgY}$ and improve its performance in the gastrointestinal tract (Zhang J et al., 2020). Immune checkpoint inhibitors (ICIs) can significantly improve the survival of some advanced malignant tumors, especially metastatic malignant melanoma and lung cancer (Afzal et al., 2018; Madden and Kasler, 2019). They include anti-cytotoxic $\mathrm{T}$ lymphocyte-associated antigen-4 (anti-CTLA-4) antibodies (ipilimumab and tremelimumab), anti-programmed cell death protein 1 (antiPD-1) antibodies (nivolumab and pembrolizumab), and anti-programmed death-ligand 1 (anti-PDL1) antibodies (atezolizumab, avelumab, and durvalumab) (Baxi et al., 2018). Previous studies have shown that ICI combination therapy, such as nivolumab plus ipilimumab, has particular efficacy in lung cancer, renal cell carcinoma, and malignant melanoma (Wolchok

$\square$ Xiahong LIN, linxiahongdr@fjmu.edu.cn

* The two authors contributed equally to this work

(iD Xiahong LIN, https://orcid.org/0000-0002-5515-730X

Received Aug. 7, 2020; Revision accepted Nov. 8, 2020; Crosschecked Jan. 6, 2021

(c) Zhejiang University Press 2021 et al., 2017; Derosa et al., 2018; Doroshow et al., 2019). However, ICIs may also lead to many immune-related adverse events (irAEs), even causing severe complications in certain cases. The most wellestablished toxicities from ICI therapy are gastrointestinal irAEs, including enteritis, enterocolitis, microscopic colitis, and gastritis, which have attracted public attention in recent years; reports of such events associated with ICI therapy also have increased (Tandon et al., 2018; de Malet et al., 2019). These gastrointestinal irAEs may generally respond well to corticosteroids and infliximab (Haanen et al., 2017). Although most of these irAEs are low-grade, a lack of detection and timely treatment may incur severe or fatal complications.

Many clinical oncologists and gastroenterologists are unfamiliar with gastrointestinal irAEs caused by ICI treatment, making it impossible to detect and deal with the resulting immunotoxicity in time. In addition, the previous scientific literature lacks comprehensive and detailed reports on gastrointestinal irAEs caused by ICIs, especially on cases of megacolon. In this study, we used the World Health Organization (WHO) global individual case safety report database, known as VigiBase (herein also referred to as Adverse Drug Reaction Database), which is managed by the Uppsala Monitoring Center in Sweden, to fully understand the gastrointestinal irAEs caused by ICI treatment, and their clinical characteristics.

The present study is a disproportionality analysis leveraging VigiBase, which includes reports from more than 130 countries, and represents over $90 \%$ of the world's population. It contains more than 19 million 
individual case safety reports (ICSRs) submitted by national pharmacovigilance centers since 1967 . These reports are generally notified post-marketing and originate from many kinds of sources, including pharmacists, healthcare professionals, patients, and physicians. Adverse drug reactions (ADRs) in VigiBase are coded according to the Medical Dictionary for Regulatory Activities (MedDRA).

In the present observational, retrospective, and disproportionate analysis, based on the VigiBase database, we report suspicious gastrointestinal irAEs related to ICI therapy from January 1, 2011 to March 6, 2019 , and describe the clinical features of cases in detail. The reference group consists of all the adverse reaction reports in VigiBase during the same study period. The main gastrointestinal irAEs are divided into 13 categories, namely enterocolitis, gastrointestinal perforation, megacolon, duodenitis, intestinal obstruction, esophagitis, diarrhea, colon operation, gastritis, inflammatory bowel disease, gastrointestinal hemorrhage, abdominal discomfort, and vomiting (Table 1).

Each adverse reaction report associated with ICI treatment includes patient characteristics (age and sex), management information (country, reporting date, and notifier), drug information (WHO drug name, indication, route of administration, dosage regimen, start and end dates), and ADR information (MedDRA classification terms, onset of ADRs, and final outcomes). The five ICI drugs reported in the study are anti-PD-L1 antibodies (atezolizumab and durvalumab), anti-CTLA-4 antibody (ipilimumab), and anti-PD-1 antibodies (nivolumab and pembrolizumab). A severe adverse event is defined as an event leading to any other medically important conditions that require hospitalization (initial or prolonged), is life-threatening, or causes death. It is worth noting that reports of ADRs were used for the purposes of this study starting from January 1, 2011.

VigiBase is known as a case-non-case analytic database; therefore, it was used for disproportionality analysis to assess whether suspected drug-induced gastrointestinal events were differentially reported between ICIs and other drugs in the full database. Gastrointestinal irAEs among different ICI regimens were also compared by disproportionality analyses, i. e., anti-PD-1 or anti-PD-L1 versus anti-CTLA-4 monotherapy, and monotherapy versus combination ICI therapy (anti-PD-1 or anti-PD-L1 plus anti-CTLA-4 combination therapy). The analyses aimed to identify adverse drug events by relative frequency, which is

Table 1 Reports of ICI-associated gastrointestinal irAEs versus those included in the full database of VigiBase (from January 1, 2011 to March 6, 2019)

\begin{tabular}{lccc}
\hline \multicolumn{1}{c}{ Gastrointestinal irAE } & $\begin{array}{c}\text { ICSRs for ICIs } \\
\text { (ICIs reports in total: 61 931) }\end{array}$ & $\begin{array}{c}\text { ICSRs in full database } \\
\text { (reports in total: 13 300 773) }\end{array}$ & $\begin{array}{c}\text { Information component } \\
(95 \% \text { CI) }\end{array}$ \\
\hline Enterocolitis & $3066(4.82 \%)$ & $23850(0.18 \%)$ & $4.80(4.75-4.85)$ \\
Gastrointestinal perforation & $380(0.40 \%)$ & $12983(0.10 \%)$ & $2.68(2.51-2.80)$ \\
Megacolon & $15(0.01 \%)$ & $535(0.004 \%)$ & $2.46(1.62-3.05)$ \\
Duodenitis & $44(0.06 \%)$ & $4358(0.03 \%)$ & $1.19(0.71-1.54)$ \\
Intestinal obstruction & $259(0.35 \%)$ & $25760(0.19 \%)$ & $1.19(0.99-1.33)$ \\
Esophagitis & $62(0.10 \%)$ & $7344(0.06 \%)$ & $0.85(0.43-1.15)$ \\
Diarrhea & $3246(5.24 \%)$ & $416055(3.13 \%)$ & $0.74(0.69-0.79)$ \\
Colon operation & $22(0.04 \%)$ & $2858(0.02 \%)$ & $0.70(-0.01-1.21)$ \\
Gastritis & $166(0.27 \%)$ & $39951(0.30 \%)$ & $-0.16(-0.42-0.02)$ \\
Inflammatory bowel disease & $122(0.20 \%)$ & $42884(0.32 \%)$ & $-0.71(-1.01-0.49)$ \\
Gastrointestinal hemorrhage & $365(0.59 \%)$ & $131244(0.99 \%)$ & $-0.74(-0.92-0.62)$ \\
Abdominal discomfort & $1136(1.83 \%)$ & $520487(3.91 \%)$ & $-1.09(-1.19-1.02)$ \\
Vomiting & $2616(4.22 \%)$ & $1248590(9.39 \%)$ & $-1.15(-1.22-1.11)$ \\
\hline
\end{tabular}

ICSRs are described as number (percentage). The information component (IC) reflects the strength of association between the specific treatment and the adverse drug event. Positive IC values are thresholds of signal detection within VigiBase. Different ICIs include ipilimumab, nivolumab, pembrolizumab, atezolizumab, durvalumab, and combination therapy (ipilimumab plus nivolumab, ipilimumab plus pembrolizumab, ipilimumab plus atezolizumab, pembrolizumab plus nivolumab, ipilimumab plus pembrolizumab plus nivolumab). All gastrointestinal irAEs were classified by group queries according to the Medical Dictionary for Regulatory Activities (MedDRA, Version 20.1). ICIs: immune checkpoint inhibitors; irAE: immune-related adverse event; ICSRs: individual case safety reports; CI: confidence interval. 
different from the overall reporting of events in the full database. The proportion of selected ADRs reported for ICIs was compared to the proportion in the full database.

Disproportionality analysis can be calculated by the information component (IC) or the reported odds ratio (ROR). The earlier can provide a conservative association metric, and reduce the risk of highlighting false associations compared to the ROR, especially for events with very low expected frequencies in the VigiBase database. Values of IC are only used to compare adverse drug events with the full database, but cannot be used for comparisons of different ICI reporting events among ICI therapies. Therefore, the IC value was used to identify meaningful ICIs that induced irAEs, while the ROR was used to compare the reporting events of ADRs in different ICI subgroups.

The value of IC, whose calculation is derived from a Bayesian confidence propagation neural network, is used to reflect the strength of the adverse drug event association (Zhao et al., 2001), which is expressed by the logarithm of prior and posterior probabilities of occurrence of ADRs. A positive IC value indicates that adverse drug events are reported more often than all reported events in the full database. The $95 \%$ confidence interval (CI) indicates the stability of IC values; the stability is higher when the 95\% CI is narrower. Disproportionality for gastrointestinal irAEs induced by ICI subgroups was analyzed by calculating the ROR $(95 \% \mathrm{CI})$. When the lower value of ROR $(95 \% \mathrm{CI})$ was larger than one, adverse reactions for the target ICI were notable ADR signals in the adverse drug event. The Bonferroni adjustment method was used to perform the comparisons among different drug treatment regimens, and the ROR CI was adjusted by using $P=0.01$. The clinical characteristics of ADRs were summarized by descriptive statistical methods, while the quantitative variables were compared by using one-way analysis of variance (ANOVA).

According to the VigiBase database, patients who received ICIs reported 61931 adverse events, whereas patients who were administered any drug reported 13300773 adverse events between January 1, 2011 and March 6, 2019. Table 1 shows the IC values for various gastrointestinal adverse events induced by ICI therapy compared to those for the same adverse event in the full database: ICI-associated enterocolitis
(3066 reports for ICIs vs. 23850 reports for the full database; IC 4.80 (95\% CI 4.75-4.85)); ICI-associated gastrointestinal perforation (380 reports for ICIs vs. 12983 reports for the full database; IC 2.68 (95\% CI 2.51-2.80)); ICI-associated duodenitis (44 reports for ICIs vs. 4358 reports for the full database; IC 1.19 (95\% CI 0.71-1.54)); ICI-associated intestinal obstruction (259 reports for ICIs vs. 25760 reports for the full database; IC 1.19 (95\% CI $0.99-1.33)$ ). It is worth noting that 15 cases of the rarely reported ICI-associated megacolon were found (535 reports for the full database; IC 2.46 (95\% CI 1.62-3.05)). All IC values were positive for the above five gastrointestinal irAEs induced by ICIs, and the values of $95 \% \mathrm{CI}$ were very narrow, which shows that these adverse events are markedly associated with ICI therapies. Thus, we focus on these five gastrointestinal irAEs to analyze differences between various ICI subgroups, and to explain the clinical characteristics of each gastrointestinal irAE.

As demonstrated in Table 2, the ROR values of the five gastrointestinal irAE were markedly different between ICI therapies, and the ROR values of duodenitis, enterocolitis, and gastrointestinal perforation were the highest in ICI combination treatments (ROR 11.68 (95\% CI 7.43-18.34), ROR 93.09 (95\% CI 85.85-100.94), and ROR 5.77 (95\% CI 3.98-8.37), respectively). We also found that the three gastrointestinal irAEs of duodenitis, enterocolitis, and intestinal obstruction were overreported for patients subjected to ICI combination treatment, versus those who received ICI monotherapy: duodenitis (ROR 8.43 (95\% CI 4.70-15.10)), enterocolitis (ROR 3.98 (95\% CI 3.64-4.35)), and intestinal obstruction (ROR 1.94 (95\% CI 1.37-2.75)). Enterocolitis, gastrointestinal perforation, and megacolon were overreported for patients who received anti-CTLA-4 treatment, versus those subjected to anti-PD-1/PD-L1 treatment (ROR 3.87 (95\% CI 3.57-4.20), ROR 5.42 (95\% CI 4.37-6.73), and ROR 4.92 (95\% CI 1.75-13.82), respectively).

Events of ICI-associated enterocolitis, gastrointestinal perforation, intestinal obstruction, and duodenitis were reported mainly from the Americas (1292/3066 (42.14\%), 193/379 (50.92\%), 138/259 (53.28\%), and 19/44 (43.18\%), respectively), while most of the megacolon cases were recorded in Europe $(5 / 15(33.33 \%))$, as shown in Table 3 . There were more male patients than female ones for the five 
Table 2 Major ICI-associated gastrointestinal ADRs compared to the full database of VigiBase (from January 1, 2011 to March 6, 2019)

\begin{tabular}{|c|c|c|c|c|c|c|c|c|c|c|}
\hline \multirow{2}{*}{ Characteristics } & \multicolumn{2}{|c|}{ Duodenitis } & \multicolumn{2}{|c|}{ Enterocolitis } & \multicolumn{2}{|c|}{$\begin{array}{c}\text { Gastrointestinal } \\
\text { perforation }\end{array}$} & \multicolumn{2}{|c|}{$\begin{array}{c}\text { Intestinal } \\
\text { obstruction }\end{array}$} & \multicolumn{2}{|c|}{ Megacolon } \\
\hline & $n / n$ & $\begin{array}{c}\text { ROR } \\
(95 \% \mathrm{CI}) \\
\end{array}$ & $n / n$ & $\begin{array}{c}\text { ROR } \\
(95 \% \mathrm{CI}) \\
\end{array}$ & $n / n$ & $\begin{array}{c}\text { ROR } \\
(95 \% \mathrm{CI}) \\
\end{array}$ & $n / n$ & $\begin{array}{c}\text { ROR } \\
(95 \% \mathrm{CI}) \\
\end{array}$ & $n / n$ & $\begin{array}{c}\text { ROR } \\
(95 \% \mathrm{CI}) \\
\end{array}$ \\
\hline $\begin{array}{l}\text { Anti-CTLA-4 vs. } \\
\text { full database }\end{array}$ & $2 / 4358$ & $\begin{array}{c}0.42 \\
(0.11-1.68)\end{array}$ & $1239 / 23850$ & $\begin{array}{c}56.67 \\
(53.42-60.11)\end{array}$ & $214 / 12983$ & $\begin{array}{c}15.97 \\
(13.96-18.27)\end{array}$ & $46 / 25760$ & $\begin{array}{c}1.75 \\
(1.32-2.32)\end{array}$ & $9 / 535$ & $\begin{array}{c}15.71 \\
(8.13-30.36)\end{array}$ \\
\hline $\begin{array}{l}\text { Anti-PD-1/ } \\
\text { Anti-PD-L1 vs. } \\
\text { full database }\end{array}$ & $25 / 4358$ & $\begin{array}{c}1.68 \\
(1.14-2.47)\end{array}$ & $1143 / 23850$ & $\begin{array}{c}14.52 \\
(13.67-15.41)\end{array}$ & $140 / 12983$ & $\begin{array}{c}2.92 \\
(2.46-3.46)\end{array}$ & $182 / 25760$ & $\begin{array}{c}2.06 \\
(1.78-2.38)\end{array}$ & $5 / 535$ & $\begin{array}{c}3.17 \\
(1.42-7.08)\end{array}$ \\
\hline $\begin{array}{l}\text { ICI combination } \\
\text { vs. full database }\end{array}$ & $17 / 4358$ & $\begin{array}{c}11.68 \\
(7.43-18.34)\end{array}$ & $684 / 23850$ & $\begin{array}{c}93.09 \\
(85.85-100.94)\end{array}$ & $26 / 12983$ & $\begin{array}{c}5.77 \\
(3.98-8.37)\end{array}$ & $31 / 25760$ & $\begin{array}{c}3.84 \\
(2.78-5.31)\end{array}$ & $1 / 535$ & $\begin{array}{c}4.98 \\
(0.70-35.40)\end{array}$ \\
\hline $\begin{array}{l}\text { Anti-CTLA-4 vs. } \\
\text { Anti-PD-1/ } \\
\text { Anti-PD-L1 }\end{array}$ & $2 / 25$ & $\begin{array}{c}0.25 \\
(0.06-1.06)\end{array}$ & $1239 / 1143$ & $\begin{array}{c}3.87 \\
(3.57-4.20)\end{array}$ & $214 / 140$ & $\begin{array}{c}5.42 \\
(4.37-6.73)\end{array}$ & $46 / 182$ & $\begin{array}{c}0.85 \\
(0.62-1.17)\end{array}$ & $9 / 5$ & $\begin{array}{c}4.92 \\
(1.75-13.82)\end{array}$ \\
\hline $\begin{array}{l}\text { ICI combination } \\
\text { vs. monotherapy }\end{array}$ & $17 / 27$ & $\begin{array}{c}8.43 \\
(4.70-15.10) \\
\end{array}$ & $684 / 2382$ & $\begin{array}{c}3.98 \\
(3.64-4.35)\end{array}$ & $26 / 354$ & $\begin{array}{c}0.98 \\
(0.67-1.44) \\
\end{array}$ & $31 / 228$ & $\begin{array}{c}1.94 \\
(1.37-2.75) \\
\end{array}$ & $1 / 14$ & $\begin{array}{c}0.83 \\
(0.11-6.25) \\
\end{array}$ \\
\hline
\end{tabular}

Anti-CTLA-4 refers to any ICSR treated with ipilimumab. Anti-PD-1/PD-L1 refers to any ICSR treated with any of the following four drugs: nivolumab, pembrolizumab, atezolizumab, and durvalumab. ICI combination refers to any ICSR treated with anti-CTLA-4 (ipilimumab) in combination with any one or two of the anti-PD-1/PD-L1, or to any ICSR treated with anti-PD-1 in combination with anti-PD-L1. Comparisons between different drug regimens were performed using the Bonferroni adjustment method. ROR confidence intervals were calculated using $P=0.01$ to adjust. ROR: reported odds ratio; CI: confidence interval; ICI: immune checkpoint inhibitor; ADR: adverse drug reaction; Anti-CTLA-4: anti-cytotoxic T lymphocyte-associated antigen-4; ICSR: individual case safety report; Anti-PD-1: anti-programmed cell death protein 1; Anti-PD-L1: anti-programmed death-ligand 1.

gastrointestinal irAEs except for megacolon (enterocolitis $60.88 \%$ vs. $39.12 \%$; gastrointestinal perforation $67.24 \%$ vs. $32.76 \%$; intestinal obstruction $60.40 \%$ vs. $39.60 \%$; duodenitis $62.79 \%$ vs. $37.21 \%$; megacolon $40.00 \%$ vs. $60.00 \%$ ). We also found that reported events of enterocolitis, duodenitis, gastrointestinal perforation, megacolon, and intestinal obstruction, associated with ICI treatment, were observed mainly in patients with lung cancer or malignant melanoma.

As shown in Table 4, reports of enterocolitis (1239 cases, 40.41\%) and gastrointestinal perforation (214 cases, 56.32\%) were the two most frequent ipilimumab-associated ADRs, while the most of intestinal obstruction cases (86 cases, 33.20\%) were nivolumab-associated, and of duodenitis cases (16 cases, 36.36\%) were ipilimumab plus nivolumab combination-associated. Megacolon was reported mainly under ipilimumab treatment (9 cases, 60.00\%). In addition, there were a small number of reports of anti-PD-L1-associated gastrointestinal irAEs (87 cases of atezolizumab and 43 cases of durvalumab). We conducted a further analysis of follow-up reports on ICI-associated gastrointestinal irAEs. It was found that 1713 cases had the final outcomes reported, including "fatal," "not recovered/not resolved," and "recovered/resolved." Most patients in the enterocolitis, intestinal obstruction, and duodenitis cases recovered or resolved after appropriate treatment, while most of the gastrointestinal perforation cases $(46.61 \%)$ had fatal consequences. The number of recovery reports of ICI-associated enterocolitis $(71.70 \%)$ was higher than that for ICI-associated gastrointestinal perforation, intestinal obstruction, duodenitis, and megacolon combined. Four megacolon cases reported final outcomes: the patient recovered in one case and did not recover in another case, while two cases had fatal consequences.

Additionally, we analyzed time to onset for ICIassociated gastrointestinal irAEs. We found that the time to onset of enterocolitis associated with antiCTLA-4 and ICI combination treatment was earlier compared to that associated with anti-PD-1/PD-L1 monotherapy (anti-CTLA-4 (55.44 \pm 3.25$)$ d, anti-PD-1/ PD-L1 (119.50 \pm 5.15$) \mathrm{d}$, and combination therapy $(58.49 \pm 4.64) \mathrm{d})$, and the time to onset of enterocolitis was not significantly different between anti-CTLA-4 and ICI combination treatments (Fig. 1a). As shown in Fig. 1b, the time to onset of gastrointestinal perforation was not significantly different between anti-CTLA-4 and anti-PD-1/PD-L1 monotherapy (anti-CTLA-4 $(58.08 \pm 6.45) \mathrm{d}$ and anti-PD-1/PD-L1 $(61.67 \pm 8.44) \mathrm{d}, P=0.7998)$. Furthermore, the time to 
Table 3 Basic information of ICI-associated gastrointestinal ADR cases reported to VigiBase (from January 1, 2011 to March 6, 2019)

\begin{tabular}{|c|c|c|c|c|c|}
\hline Basic information & $\begin{array}{c}\text { Enterocolitis } \\
(n=3066)\end{array}$ & $\begin{array}{c}\text { Gastrointestinal perforation } \\
(n=379)\end{array}$ & $\begin{array}{c}\text { Intestinal obstruction } \\
(n=259)\end{array}$ & $\begin{array}{c}\text { Duodenitis } \\
(n=44)\end{array}$ & $\begin{array}{l}\text { Megacolon } \\
(n=15)\end{array}$ \\
\hline \multicolumn{6}{|l|}{ Year } \\
\hline 2011 & $23(0.75 \%)$ & $8(2.11 \%)$ & $4(1.54 \%)$ & 0 & 0 \\
\hline 2012 & $84(2.74 \%)$ & $28(7.39 \%)$ & $9(3.47 \%)$ & 0 & $3(20.00 \%)$ \\
\hline 2013 & $103(3.36 \%)$ & $20(5.28 \%)$ & $2(0.77 \%)$ & 0 & $2(13.33 \%)$ \\
\hline 2014 & $233(7.60 \%)$ & $49(12.93 \%)$ & $10(3.86 \%)$ & $2(4.55 \%)$ & 0 \\
\hline 2015 & $369(12.04 \%)$ & $79(20.84 \%)$ & $28(10.81 \%)$ & $5(11.36 \%)$ & $3(20.00 \%)$ \\
\hline 2016 & $425(13.86 \%)$ & $45(11.87 \%)$ & $30(11.58 \%)$ & $7(15.91 \%)$ & $1(6.67 \%)$ \\
\hline 2017 & $754(24.59 \%)$ & $55(14.51 \%)$ & $62(23.94 \%)$ & $10(22.73 \%)$ & $6(40.00 \%)$ \\
\hline 2018 & $934(30.46 \%)$ & $88(23.22 \%)$ & $98(37.84 \%)$ & $15(34.09 \%)$ & 0 \\
\hline 2019 & $141(4.60 \%)$ & $7(1.85 \%)$ & $16(6.18 \%)$ & $5(11.36 \%)$ & 0 \\
\hline \multicolumn{6}{|l|}{ Gender } \\
\hline Female & $1080 / 2761(39.12 \%)$ & $115 / 351(32.76 \%)$ & $99 / 250(39.60 \%)$ & $16 / 43(37.21 \%)$ & $9 / 15(60.00 \%)$ \\
\hline Male & $1681 / 2761(60.88 \%)$ & $236 / 351(67.24 \%)$ & $151 / 250(60.40 \%)$ & $27 / 43(62.79 \%)$ & $6 / 15(40.00 \%)$ \\
\hline \multicolumn{6}{|l|}{ Age (year) } \\
\hline $0-17$ & $3 / 2173(0.14 \%)$ & 0 & 0 & 0 & 0 \\
\hline $18-44$ & $218 / 2173(10.03 \%)$ & $16 / 290(5.52 \%)$ & $14 / 205(6.83 \%)$ & $5 / 36(13.89 \%)$ & 1/13 (7.69\%) \\
\hline $45-65$ & $870 / 2173(40.04 \%)$ & $117 / 290(40.34 \%)$ & $109 / 205(53.17 \%)$ & $16 / 36(44.44 \%)$ & $6 / 13(46.15 \%)$ \\
\hline$>65$ & $1082 / 2173(49.79 \%)$ & $157 / 290(54.14 \%)$ & $82 / 205(40.00 \%)$ & $15 / 36(41.67 \%)$ & $6 / 13(46.15 \%)$ \\
\hline \multicolumn{6}{|l|}{ Region } \\
\hline Africa & $3 / 3066(0.10 \%)$ & 0 & $1 / 259(0.39 \%)$ & 0 & 0 \\
\hline Americas & $1292 / 3066(42.14 \%)$ & $193 / 379(50.92 \%)$ & $138 / 259(53.28 \%)$ & $19 / 44(43.18 \%)$ & $4 / 15(26.67 \%)$ \\
\hline Asia & 403/3066 (13.14\%) & $65 / 379(17.15 \%)$ & $61 / 259(23.55 \%)$ & $9 / 44(20.45 \%)$ & $4 / 15(26.67 \%)$ \\
\hline Europe & $1198 / 3066(39.07 \%)$ & $109 / 379(28.76 \%)$ & $51 / 259(19.69 \%)$ & $15 / 44(34.09 \%)$ & $5 / 15(33.33 \%)$ \\
\hline Oceania & $170 / 3066(5.54 \%)$ & $12 / 379(3.17 \%)$ & $8 / 259(3.09 \%)$ & $1 / 44(2.27 \%)$ & $2 / 15(13.33 \%)$ \\
\hline \multicolumn{6}{|l|}{ Notifier $^{\mathrm{a}}$} \\
\hline Notifier1 & $2391 / 2912(82.11 \%)$ & $289 / 366(78.96 \%)$ & $205 / 248(82.66 \%)$ & $40 / 42(95.24 \%)$ & $9 / 14(64.29 \%)$ \\
\hline Notifier2 & $521 / 2912(17.89 \%)$ & $77 / 366(21.04 \%)$ & $43 / 248(17.34 \%)$ & $2 / 42(4.76 \%)$ & $5 / 14(35.71 \%)$ \\
\hline \multicolumn{6}{|l|}{ Indication $^{\mathrm{b}}$} \\
\hline $\begin{array}{l}\text { Malignant } \\
\text { melanoma }\end{array}$ & $1533 / 2350(65.23 \%)$ & $168 / 277(60.65 \%)$ & $67 / 180(37.22 \%)$ & $14 / 34(41.18 \%)$ & $8 / 10(80.00 \%)$ \\
\hline Lung cancer & $608 / 2350(25.87 \%)$ & 78/277 (28.16\%) & $53 / 180(29.44 \%)$ & $11 / 34(32.35 \%)$ & $2 / 10(20.00 \%)$ \\
\hline Kidney cancer & $83 / 2350(3.53 \%)$ & 4/277 (1.44\%) & $9 / 180(5.00 \%)$ & $4 / 34(11.76 \%)$ & 0 \\
\hline Bladder cancer & $44 / 2350(1.87 \%)$ & $10 / 277(3.61 \%)$ & $29 / 180(16.11 \%)$ & $1 / 34(2.94 \%)$ & 0 \\
\hline Gastric cancer & $12 / 2350(0.51 \%)$ & $8 / 277(2.89 \%)$ & $8 / 180(4.44 \%)$ & 0 & 0 \\
\hline Prostate cancer & $20 / 2350(0.85 \%)$ & $3 / 277(1.08 \%)$ & $2 / 180(1.11 \%)$ & 0 & 0 \\
\hline Colon cancer & $13 / 2350(0.55 \%)$ & $1 / 277(0.36 \%)$ & $8 / 180(4.44 \%)$ & $2 / 34(5.88 \%)$ & 0 \\
\hline
\end{tabular}

Data are expressed as number (percentage). ${ }^{a}$ Notifier1 refers to physician, pharmacist, or other healthcare professional; Notifier 2 refers to lawyer or consumer. ${ }^{b}$ Indication refers to the primary tumors treated by ICIs. ICI: immune checkpoint inhibitor; ADR: adverse drug reaction.

onset of intestinal obstruction, as shown in Fig. 1c, was not significantly different between anti-CTLA-4 and anti-PD-1/PD-L1 monotherapy (anti-CTLA-4

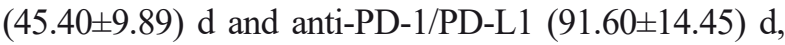
$P=0.1760)$.

Early clinical outcomes with ICI therapies have indicated that ICIs can improve patient survival; therefore, such therapies have been widely used to treat many malignant tumors, such as malignant melanoma (Wolchok et al., 2017; Afzal et al., 2018), renal cell carcinoma (Derosa et al., 2018), lung cancer (Doroshow et al., 2019; Madden and Kasler, 2019), and urothelial cancer (Rodriguez-Vida et al., 2018). Meanwhile, ICIs can also disturb the maintenance of patient 
Table 4 Comparison of ICI regimen for ICI-associated digestive system ADRs reported to VigiBase (from January 1, 2011 to March 6, 2019)

\begin{tabular}{|c|c|c|c|c|c|}
\hline ICI regimen/outcome & $\begin{array}{l}\text { Enterocolitis } \\
\qquad(n=3066)\end{array}$ & $\begin{array}{c}\text { Gastrointestinal } \\
\text { perforation } \\
(n=380)\end{array}$ & $\begin{array}{c}\text { Intestinal } \\
\text { obstruction } \\
(n=259)\end{array}$ & $\begin{array}{c}\text { Duodenitis } \\
\quad(n=44)\end{array}$ & $\begin{array}{l}\text { Megacolon } \\
\quad(n=15)\end{array}$ \\
\hline \multicolumn{6}{|l|}{ Drug group } \\
\hline Ipilimumab & $1239(40.41 \%)$ & $214(56.32 \%)$ & $46(17.76 \%)$ & $2(4.55 \%)$ & $9(60.00 \%)$ \\
\hline Nivolumab & $660(21.53 \%)$ & $67(17.63 \%)$ & $86(33.20 \%)$ & $11(25.00 \%)$ & $5(33.33 \%)$ \\
\hline Pembrolizumab & $406(13.24 \%)$ & $60(15.79 \%)$ & $58(22.39 \%)$ & $12(27.27 \%)$ & 0 \\
\hline Atezolizumab & $46(1.50 \%)$ & $10(2.63 \%)$ & $30(11.58 \%)$ & $1(2.27 \%)$ & 0 \\
\hline Durvalumab & $31(1.01 \%)$ & $3(0.79 \%)$ & $8(3.09 \%)$ & $1(2.27 \%)$ & 0 \\
\hline Ipilimumab plus nivolumab & $656(21.40 \%)$ & $25(6.58)$ & $29(11.20 \%)$ & $16(36.36 \%)$ & $1(6.67 \%)$ \\
\hline Ipilimumab plus pembrolizumab & $19(0.62 \%)$ & $1(0.26 \%)$ & $1(0.39 \%)$ & $1(2.27 \%)$ & 0 \\
\hline Ipilimumab plus atezolizumab & $1(0.03 \%)$ & 0 & 0 & 0 & 0 \\
\hline Pembrolizumab plus nivolumab & $1(0.03 \%)$ & 0 & 0 & 0 & 0 \\
\hline $\begin{array}{l}\text { Ipilimumab plus pembrolizumab } \\
\text { plus nivolumab }\end{array}$ & $7(0.23 \%)$ & 0 & $1(0.39 \%)$ & 0 & 0 \\
\hline \multicolumn{6}{|l|}{ Drug doses } \\
\hline \multicolumn{6}{|l|}{ Monotherapy with ipilimumab } \\
\hline $3 \mathrm{mg} / \mathrm{kg}$ & $392 / 742(52.83 \%)$ & $61 / 136(44.85 \%)$ & $7 / 26(26.92 \%)$ & 0 & $2 / 7(28.57 \%)$ \\
\hline $10 \mathrm{mg} / \mathrm{kg}$ & $120 / 742(16.17 \%)$ & $22 / 136(16.18 \%)$ & $9 / 26(34.62 \%)$ & $1 / 1(100.00 \%)$ & $1 / 7(14.29 \%)$ \\
\hline$<500 \mathrm{mg}$ & $156 / 742(21.02 \%)$ & $43 / 136(31.62 \%)$ & $3 / 26(11.54 \%)$ & 0 & $4 / 7(57.14 \%)$ \\
\hline $500-1000 \mathrm{mg}$ & $47 / 742(6.33 \%)$ & $7 / 136(5.15 \%)$ & $4 / 26(15.38 \%)$ & 0 & 0 \\
\hline$>1000 \mathrm{mg}$ & $27 / 742(3.64 \%)$ & $3 / 136(2.21 \%)$ & $3 / 26(11.54 \%)$ & 0 & 0 \\
\hline \multicolumn{6}{|l|}{ Monotherapy with nivolumab } \\
\hline $1-2 \mathrm{mg} / \mathrm{kg}$ & $13 / 408(3.19 \%)$ & $5 / 53(9.43 \%)$ & $5 / 69(7.25 \%)$ & 0 & 0 \\
\hline$\geqslant 3 \mathrm{mg} / \mathrm{kg}$ & $246 / 408(60.29 \%)$ & $26 / 53(49.06 \%)$ & $38 / 69(55.07 \%)$ & $4 / 8(50.00 \%)$ & 0 \\
\hline$\leqslant 240 \mathrm{mg}$ & $112 / 408(27.45 \%)$ & $19 / 53(35.85 \%)$ & $21 / 69(30.43 \%)$ & $4 / 8(50.00 \%)$ & $3 / 3(100.00 \%)$ \\
\hline $240-480 \mathrm{mg}$ & $37 / 408(9.07 \%)$ & $3 / 53(5.66 \%)$ & $5 / 69(7.25 \%)$ & 0 & 0 \\
\hline \multicolumn{6}{|l|}{ Monotherapy with pembrolizumab } \\
\hline $2 \mathrm{mg} / \mathrm{kg}$ & $61 / 247(24.70 \%)$ & $4 / 45(8.89 \%)$ & $7 / 44(15.91 \%)$ & $1 / 9(11.11 \%)$ & 0 \\
\hline$\leqslant 200 \mathrm{mg}$ & $186 / 247(75.30 \%)$ & $41 / 45(91.11 \%)$ & $37 / 44(84.09 \%)$ & $8 / 9(88.89 \%)$ & 0 \\
\hline \multicolumn{6}{|l|}{ Combination therapy } \\
\hline $\begin{array}{l}\text { Ipilimumab }(3 \mathrm{mg} / \mathrm{kg}) \text { plus } \\
\text { nivolumab }(1-2 \mathrm{mg} / \mathrm{kg})\end{array}$ & $150 / 367(40.87 \%)$ & $6 / 9(66.67 \%)$ & $8 / 17(47.06 \%)$ & $2 / 7(28.57 \%)$ & 0 \\
\hline $\begin{array}{l}\text { Ipilimumab (3 mg/kg) plus } \\
\text { nivolumab }(\geqslant 3 \mathrm{mg} / \mathrm{kg})\end{array}$ & $86 / 367(23.43 \%)$ & $2 / 9(22.22 \%)$ & $5 / 17(29.41 \%)$ & $2 / 7(28.57 \%)$ & 0 \\
\hline $\begin{array}{l}\text { Ipilimumab }(\leqslant 500 \mathrm{mg}) \text { plus } \\
\text { nivolumab }(\leqslant 240 \mathrm{mg})\end{array}$ & $124 / 367(33.78 \%)$ & $1 / 9(11.11 \%)$ & $3 / 17(17.65 \%)$ & $2 / 7(28.57 \%)$ & 0 \\
\hline $\begin{array}{l}\text { Ipilimumab }(\leqslant 500 \mathrm{mg}) \text { plus } \\
\text { nivolumab }(>240 \mathrm{mg})\end{array}$ & $7 / 367(1.91 \%)$ & 0 & $1 / 17(5.88 \%)$ & $1 / 7(14.29 \%)$ & 0 \\
\hline \multicolumn{6}{|l|}{ Outcome } \\
\hline Recovered/resolved & $960 / 1339(71.70 \%)$ & $82 / 221(37.10 \%)$ & $73 / 134(54.48 \%)$ & $8 / 15(53.33 \%)$ & $1 / 4(25.00 \%)$ \\
\hline Not recovered/not resolved & $252 / 1339(18.82 \%)$ & $36 / 221(16.29 \%)$ & $35 / 134(26.12 \%)$ & $7 / 15(46.67 \%)$ & $1 / 4(25.00 \%)$ \\
\hline Fatal & $127 / 1339(9.48 \%)$ & $103 / 221(46.61 \%)$ & $26 / 134(19.40 \%)$ & 0 & $2 / 4(50.00 \%)$ \\
\hline
\end{tabular}

Data are expressed as number (percentage). Drug doses are expressed in VigiBase in two forms: $\mathrm{mg} / \mathrm{kg}$ and total dose. Drug doses are grouped according to dosage and administration recommended in the drug prescription information. Recommended dosage for nivolumab: $3 \mathrm{mg} / \mathrm{kg}$ every 2 weeks, $240 \mathrm{mg}$ every 2 weeks, or $480 \mathrm{mg}$ every 4 weeks for different cancers. Recommended dosage for pembrolizumab: $200 \mathrm{mg}$ every 3 weeks, $2 \mathrm{mg} / \mathrm{kg}$ every 3 weeks for different cancers. Recommended dosage for ipilimumab: $3 \mathrm{mg} / \mathrm{kg}$ every 3 weeks or $10 \mathrm{mg} / \mathrm{kg}$ every 3 weeks for different cancers. Recommended dosage for combined ICI therapy: $3 \mathrm{mg} / \mathrm{kg}$ nivolumab followed by $1 \mathrm{mg} / \mathrm{kg}$ Ipilimumab on the same day every 3 weeks for 4 doses, and then $240 \mathrm{mg}$ nivolumab every 2 weeks or $480 \mathrm{mg}$ every 4 weeks. ICI: immune checkpoint inhibitor; ADR: adverse drug reaction.

immune homeostasis, as they suppress coinhibitory molecules between T-cells and other immune cells, which mediate the tumor killing process (Sangro et al.,
2020). In addition, ICIs have side effects and toxicities in the process of tumor therapy, commonly referred to as irAEs. Therapies involving ICIs, such 
(a)

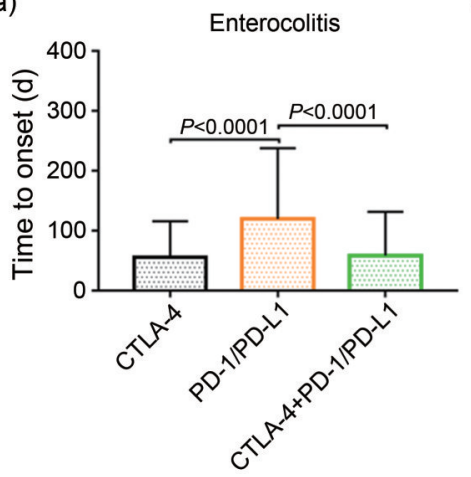

(b)

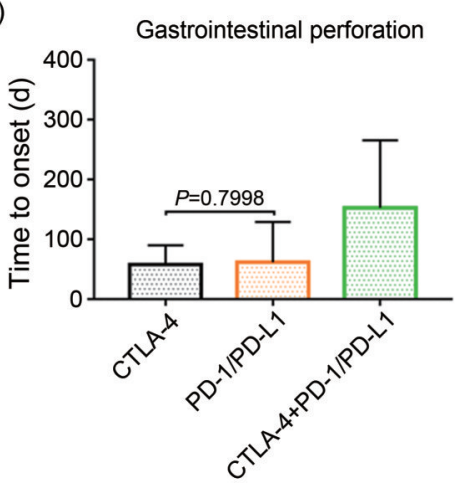

(c)

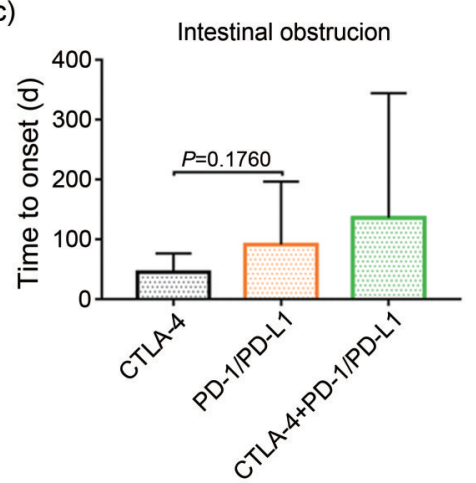

Fig. 1 Time to onset of ICI-associated gastrointestinal irAEs reported to VigiBase (from January 1, 2011 to March 6, 2019). (a) ICI-associated enterocolitis ( $n=1145)$; (b) ICI-associated gastrointestinal perforation ( $n=113)$; (c) ICIassociated intestinal obstruction $(n=86)$. Date are expressed as mean \pm standard deviation. ICI: immune checkpoint inhibitor; irAE: immune-related adverse event; CTLA-4: cytotoxic T lymphocyte-associated antigen-4; PD-1: programmed cell death protein 1; PD-L1: programmed death-ligand 1.

as anti-PD-1, anti-PD-L1, or anti-CTLA-4 antibodies, have been proved to effectively treat metastatic malignant melanoma and lung cancer (Afzal et al., 2018; Madden and Kasler, 2019). However, they cause gastrointestinal irAEs, including enteritis, enterocolitis, microscopic colitis, and gastritis (Tandon et al., 2018; de Malet et al., 2019). Additionally, certain severe or fatal irAEs were indicated in a previous study, including gastrointestinal perforation and intestinal obstruction associated with ICI therapy (Abu-Sbeih and Wang, 2020).

Thus far, the present study includes the largest and most extensive database of the clinical characteristics of ICI-associated gastrointestinal irAEs, acquired by the analysis of individual case safety reports from the WHO pharmacovigilance database. Consistently with previous studies, our results indicate that ICI-associated enterocolitis, duodenitis, gastrointestinal perforation, and intestinal obstruction are dramatically increased, and the associated risk posed by ICI combination treatment may be higher than that by ICI monotherapy. In addition, 15 cases of the rarely reported ICI-associated megacolon are included herein, which is infrequent in the clinical setting.

Previous research has shown that gastrointestinal irAEs are closely related to anti-CTLA-4 or anti-PD-1/ PD-L1 therapy (Xu et al., 2018). It was observed in the present study that, compared to anti-PD-1/PD-L1 therapy, patients receiving anti-CTLA-4 therapy may have a higher risk of developing enterocolitis (ROR 3.87 (95\% CI 3.57-4.20)), gastrointestinal perforation (ROR 5.42 (95\% CI 4.37-6.73)), and megacolon
(ROR 4.92 (95\% CI 1.75-13.82)). The combined ICI therapy of anti-CTLA-4 and anti-PD-1/PD-L1 has been suggested as highly efficient in treating malignant tumors (Yuan et al., 2019). Combined ICI therapy, however, may result in more severe immune-related ADRs (Gu et al., 2019). Our study indicates that, in agreement with previous research, patients receiving combined ICI therapy may have a higher risk of developing gastrointestinal irAEs compared to ICI monotherapy, including duodenitis (ROR 8.43 (95\% CI 4.70-15.10)), enterocolitis (ROR 3.98 (95\% CI 3.64-4.35)), and intestinal obstruction (ROR 1.94 (95\% CI 1.37-2.75)).

In the current study, it is demonstrated that most of the gastrointestinal irAEs occurred in patients with melanoma and lung cancer, with more cases in males than females, except for megacolon. Most of the gastrointestinal irAEs occurred within six weeks, and immune-related enterocolitis in ICI combination therapy manifested earlier than those in anti-PD-1/PD-L1 treatment, which was consistent with previous research (Wang et al., 2018). Additionally, it is indicated that anti-CTLA-4 therapy had a dramatically earlier time to onset of enterocolitis than anti-PD-1/PD-L1 therapy, but no significant difference between the onset time of gastrointestinal perforation and intestinal obstruction was observed.

We also analyzed cases of ICI-associated megacolon, which were very rare in the clinical setting, and had only been recorded as case reports in previous research (Cho and Choi, 2020). Fifteen megacolon cases are featured herein, most of which involved 
patients with lung cancer or malignant melanoma, and occurred in anti-CTLA-4 therapy (ipilimumab). Interestingly, ICI-associated megacolon was mainly reported from Europe but not Americas. The possible reason is that megacolon is commonly named grade 4 colitis in Americas, and has been described similarly in previous research (Wang et al., 2017). Clinically toxic megacolon requires rapid multidisciplinary assessment, and individual treatments need to be developed based on the underlying causes (Leppkes et al., 2015). If the cause is likely an infection or inflammation, perioperative morbidity and mortality can be reduced by conservative treatment. The effect of treatment needs to be re-evaluated after $72 \mathrm{~h}$ and after $7 \mathrm{~d}$ to further ensure patient safety. Thus, the toxic megacolon adverse event needs to be closely monitored during treatment by ICIs, especially by anti-CTLA-4. The risk factors of ICI-related megacolon are not yet clear, and hence they need to be investigated in future clinical trials. It was observed in our study that most patients of the enterocolitis, intestinal obstruction, and duodenitis cases were recovered or resolved after precise treatment, while most of the gastrointestinal perforation cases had fatal consequences. The number of recovery reports for ICIassociated enterocolitis was found to be larger compared to ICI-associated gastrointestinal perforation, intestinal obstruction, duodenitis, and megacolon combined. Therefore, ICI-related enterocolitis is treated conservatively with most patients capable of recovery, and there is usually no need to discontinue the drug (Zhang ML et al., 2020). Additionally, one of the most common drug side effects, esophagitis, was absent in gastrointestinal irAEs of our comprehensive assessment, demonstrating that the esophagus is likely not involved in gastrointestinal irAEs associated with ICIs therapy. Thus, it seems fortunate that the esophagus, an integral section of the gastrointestinal tract, appears to be spared in the procession of ICI therapy.

The VigiBase database has some limitations, which are also important to mention for the scope of this study. First, detailed information on clinical data, which may help to better evaluate patient responses related to irAEs, was missing from the VigiBase database. Second, when a report involved several drugs and/or several adverse events, we treated the adverse drug event pair as the basic unit rather than the reported event; the results of this pharmacovigilance analysis may thus be biased. Third, case reports of gastrointestinal disease associated with ICI therapies may not have been submitted to the VigiBase database, leading to a further bias in the database. Finally, the IC value does not indicate a causal relationship between ICIs and irAEs, but shows the related dependence of the ICI drugs and adverse event reports. In contrast to existing studies, however, the intensity of the large number of records at the national level supports our research aimed at quantifying the potential risks, but the true risks of these adverse events should be determined in prospective studies. Hence, a complete understanding of the limitations of VigiBase can help us better analyze ADRs, and produce useful clinical research hypotheses.

The present study shows that the number of gastrointestinal irAEs, including enterocolitis, gastrointestinal perforation, duodenitis, and intestinal obstruction cases, has been rising dramatically, with several reported events of megacolon, which is rare in the clinical setting. Most gastrointestinal irAEs occur early during the course of ICI therapy. Consequently, it is important to raise an awareness of gastrointestinal irAEs associated with treatment by the ICI therapy of some advanced malignant tumors.

\section{Acknowledgments}

The supplied data come from various sources from VigiBase, and the probability that the suspected adverse effect is drug-related is not the same in all cases. The information does not represent the opinion of the Uppsala Monitoring Center or the WHO. Camilla Westerberg of the custom search team at the Uppsala Monitoring Centre (Uppsala, Sweden) research department is thanked for his help with the data extraction.

\section{Author contributions}

The study design was created by Xiahong LIN. Data collection and analysis was performed by Sifu HUANG, Xuefeng BAI, and Kainan ZHENG. Taiyong FANG and Yanta GUO conducted the literature search. Sifu HUANG and Xuefeng BAI wrote and edited the manuscript, and they contributed equally to this study. Xiahong LIN, Sifu HUANG, and Xuefeng BAI were responsible for the final modification of the manuscript. All authors have read and approved the final version of the manuscript.

\section{Compliance with ethics guidelines}

Sifu HUANG, Xuefeng BAI, Taiyong FANG, Yanta GUO, Kainan ZHENG, and Xiahong LIN declare that they have no conflicts of interest.

This article does not contain any studies with human or animal subjects performed by any of the authors. 


\section{References}

Abu-Sbeih H, Wang YH, 2020. Gastrointestinal tract adverse events. In: Naing A, Hajjar J (Eds.), Immunotherapy. Springer, Cham, p.247-253. https://doi.org/10.1007/978-3-030-41008-7_12

Afzal MZ, Mercado RR, Shirai K, 2018. Efficacy of metformin in combination with immune checkpoint inhibitors (antiPD-1/anti-CTLA-4) in metastatic malignant melanoma. $J$ Immunother Cancer, 6:64. https://doi.org/10.1186/s40425-018-0375-1

Baxi S, Yang A, Gennarelli RL, et al., 2018. Immune-related adverse events for anti-PD-1 and anti-PD-L1 drugs: systematic review and meta-analysis. BMJ, 360:k793. https://doi.org/10.1136/bmj.k793

Cho JH, Choi JH, 2020. Cytomegalovirus ileo-pancolitis presenting as toxic megacolon in an immunocompetent patient: a case report. World J Clin Cases, 8(3):552-559. https://doi.org/10.12998/wjcc.v8.i3.552

de Malet A, Antoni G, Collins M, et al., 2019. Evolution and recurrence of gastrointestinal immune-related adverse events induced by immune checkpoint inhibitors. Eur $J$ Cancer, 106:106-114. https://doi.org/10.1016/j.ejca.2018.10.006

Derosa L, Hellmann MD, Spaziano M, et al., 2018. Negative association of antibiotics on clinical activity of immune checkpoint inhibitors in patients with advanced renal cell and non-small-cell lung cancer. Ann Oncol, 29(6): 1437-1444. https://doi.org/10.1093/annonc/mdy103

Doroshow DB, Sanmamed MF, Hastings K, et al., 2019. Immunotherapy in non-small cell lung cancer: facts and hopes. Clin Cancer Res, 25(15):4592-4602. https://doi.org/10.1158/1078-0432.CCR-18-1538

Gu LH, Khadaroo PA, Su H, et al., 2019. The safety and tolerability of combined immune checkpoint inhibitors (anti-PD-1/PD-L1 plus anti-CTLA-4): a systematic review and meta-analysis. BMC Cancer, 19:559. https://doi.org/10.1186/s12885-019-5785-Z

Haanen JBAG, Carbonnel F, Robert C, et al., 2017. Management of toxicities from immunotherapy: ESMO Clinical Practice Guidelines for diagnosis, treatment and follow-up. Ann Oncol, 28(Suppl_4):iv119-iv142. https://doi.org/10.1093/annonc/mdx225

Leppkes M, Ganslmayer M, Strauß R, et al., 2015. Das toxic megacolon. Med Klin Intensivmed Notfmed, 110(7):500-505. https://doi.org/10.1007/s00063-015-0076-1

Madden K, Kasler MK, 2019. Immune checkpoint inhibitors in lung cancer and melanoma. Semin Oncol Nurs, 35(5):150932. https://doi.org/10.1016/j.soncn.2019.08.011

Rodriguez-Vida A, Perez-Gracia JL, Bellmunt J, 2018. Immunotherapy combinations and sequences in urothelial cancer: facts and hopes. Clin Cancer Res, 24(24):6115-6124. https://doi.org/10.1158/1078-0432.CCR-17-3108
Sangro B, Chan SL, Meyer T, et al., 2020. Diagnosis and management of toxicities of immune checkpoint inhibitors in hepatocellular carcinoma. J Hepatol, 72(2):320-341. https://doi.org/10.1016/j.jhep.2019.10.021

Song M, Li ZL, Zhou YJ, et al., 2020. Gastrointestinal involvement of COVID-19 and potential faecal transmission of SARS-CoV-2. J Zhejiang Univ-Sci B (Biomed \& Biotechnol), 21(9):749-751. https://doi.org/10.1631/jzus.B2000253

Tandon P, Bourassa-Blanchette S, Bishay K, et al., 2018. The risk of diarrhea and colitis in patients with advanced melanoma undergoing immune checkpoint inhibitor therapy: a systematic review and meta-analysis. J Immunother, 41(3): 101-108. https://doi.org/10.1097/CJI.0000000000000213

Wang DY, Ye F, Zhao SL, et al., 2017. Incidence of immune checkpoint inhibitor-related colitis in solid tumor patients: a systematic review and meta-analysis. Oncoimmunology, 6(10):e1344805. https://doi.org/10.1080/2162402X.2017.1344805

Wang DY, Salem JE, Cohen JV, et al., 2018. Fatal toxic effects associated with immune checkpoint inhibitors: a systematic review and meta-analysis. JAMA Oncol, 4(12):1721-1728. https://doi.org/10.1001/jamaoncol.2018.3923

Wolchok JD, Chiarion-Sileni V, Gonzalez R, et al., 2017. Overall survival with combined nivolumab and ipilimumab in advanced melanoma. N Engl J Med, 377(14):1345-1356. https://doi.org/10.1056/NEJMoa1709684

Xu C, Chen YP, Du XJ, et al., 2018. Comparative safety of immune checkpoint inhibitors in cancer: systematic review and network meta-analysis. $B M J, 363: \mathrm{k} 4226$. https://doi.org/10.1136/bmj.k4226

Yuan ZH, Yang HK, Wei YM, 2019. Combined induction with anti-PD-1 and anti-CTLA-4 antibodies provides synergistic antitumor effects in DC-CIK cells in renal carcinoma cell lines. Int J Clin Exp Pathol, 12(1):123-132.

Zhang J, Li HH, Chen YF, et al., 2020. Microencapsulation of immunoglobulin Y: optimization with response surface morphology and controlled release during simulated gastrointestinal digestion. $J$ Zhejiang Univ-Sci B (Biomed \& Biotechnol), 21(8):611-627. https://doi.org/10.1631/jzus.B2000172

Zhang ML, Neyaz A, Patil D, et al., 2020. Immune-related adverse events in the gastrointestinal tract: diagnostic utility of upper gastrointestinal biopsies. Histopathology, 76(2):233-243. https://doi.org/10.1111/his.13963

Zhao SZ, Reynolds MW, Lefkowith J, et al., 2001. A comparison of renal-related adverse drug reactions between rofecoxib and celecoxib, based on the World Health Organization/Uppsala Monitoring Centre safety database. Clin Ther, 23(9):1478-1491. https://doi.org/10.1016/s0149-2918(01)80121-1 